\title{
TAK1 suppresses RIPK1-dependent cell death and is associated with disease progression in melanoma
}

\author{
Biswajit Podder ${ }^{1,2}$ - Cristiano Guttà ${ }^{1} \cdot$ Jan Rožanc ${ }^{3}$ - Elke Gerlach ${ }^{1}$ - Maria Feoktistova ${ }^{2}$. \\ Diana Panayotova-Dimitrova ${ }^{2} \cdot$ Leonidas G. Alexopoulos $^{3,4} \cdot$ Martin Leverkus $^{2} \cdot$ Markus Rehm $_{(1)}^{1,5,6,7}$
}

Received: 21 December 2018 / Revised: 28 January 2019 / Accepted: 12 February 2019 / Published online: 8 March 2019

(c) ADMC Associazione Differenziamento e Morte Cellulare 2019

\begin{abstract}
Melanoma cells are highly resistant to conventional genotoxic agents, and BRAFV600/MEK-targeted therapies as well as immunotherapies frequently remain inefficient. Alternative means to treat melanoma, in particular through the induction of programmed cell death modalities such as apoptosis or necroptosis, therefore still need to be explored. Here, we report that melanoma cell lines expressing notable amounts of RIPK1, RIPK3 and MLKL, the key players of necroptosis signal transduction, fail to execute necroptotic cell death. Interestingly, the activity of transforming growth factor $\beta$-activated kinase 1 (TAK1) appears to prevent RIPK1 from contributing to cell death induction, since TAK1 inhibition by (5Z)-7-Oxozeaenol, deletion of MAP $3 K 7$ or the expression of inactive TAK1 were sufficient to sensitize melanoma cells to RIPK1-dependent cell death in response to TNF $\alpha$ or TRAIL based combination treatments. However, cell death was executed exclusively by apoptosis, even when RIPK3 expression was high. In addition, TAK1 inhibitor (5Z)-7-Oxozeaenol suppressed intrinsic or treatment-induced pro-survival signaling as well as the secretion of cytokines and soluble factors associated with melanoma disease progression. Correspondingly, elevated expression of TAK1 correlates with reduced disease free survival in patients diagnosed with primary melanoma. Overall, our results therefore demonstrate that TAK1 suppresses the susceptibility to RIPK1-dependent cell death and that high expression of TAK1 indicates an increased risk for disease progression in melanoma.
\end{abstract}

These authors contributed equally: Martin Leverkus, Markus Rehm

Edited by M. Piacentini

Supplementary information The online version of this article (https:// doi.org/10.1038/s41418-019-0315-8) contains supplementary material, which is available to authorized users.

Markus Rehm

markus.morrison@izi.uni-stuttgart.de

1 Institute of Cell Biology and Immunology, University of Stuttgart, Stuttgart, Germany

2 Department for Dermatology and Allergology, University Hospital Aachen, RWTH Aachen, Aachen, Germany

3 ProtATonce Ltd, Science Park Demokritos, Athens, Greece

4 Department of Mechanical Engineering, National Technical Univerisity of Athens, Athens, Greece

5 Stuttgart Research Center Systems Biology, University of Stuttgart, Stuttgart, Germany

6 Department of Physiology and Medical Physics, Royal College of Surgeons in Ireland, Dublin, Ireland

7 Centre for Systems Medicine, Royal College of Surgeons in Ireland, Dublin, Ireland

\section{Introduction}

Melanoma are highly chemoresistant and, despite the success of BRAF/MEK-targeting therapeutics and immune checkpoint inhibitor-based treatments, many patients still do not benefit from these novel treatment options or experience disease relapse $[1,2]$. An attractive addition to the armament against malignant melanoma could be treatments that induce programmed cell death through receptors of the tumor necrosis factor (TNF) family. TNF receptor activation can trigger two signaling cascades towards cell death, namely apoptosis and necroptosis [3, 4]. Apoptosis relies on the FADD-dependent activation of caspase- 8 at the death inducing signaling complex, the subsequent permeabilisation of the outer mitochondrial membrane and the activation of the apoptosis execution network [5]. Necroptosis likewise is FADD-dependent in this context but, in contrast to protease-driven apoptosis, is transduced by a kinase signaling cascade in which activating (auto-)phosphorylation of RIPK1, RIPK3 and MLKL culminates in the latter forming lytic pores in the plasma membrane [6, 7]. 
Apoptosis and necroptosis signaling branches exert interplay, with caspase- 8 proteolytically inactivating RIPK1 and RIPK3, and RIPK1 modulating apoptosis and necroptosis susceptibility depending on its activity, phosphorylation, and ubiquitylation status [3, 8, 9].

If not inducing cell death, the activation of TNF receptors and tumor necrosis factor-related apoptosis-inducing ligand (TRAIL) receptors stimulates pro-survival signaling $[3,4]$. For example, tumor-intrinsic $\mathrm{TNF} \alpha$ contributes to resistance against BRAF(V600) inhibitor-based treatments and suppresses tumor-directed immune responses [10, 11]. This highlights the need to combine TNF receptor ligands with therapeutics that reliably direct signaling towards programmed cell death. A major component of the prosurvival signaling complexes that form prior to apoptosisor necroptosis-inducing platforms are cellular inhibitor of apoptosis proteins (cIAPs) 1 and 2. cIAPs recruit the linear ubiquitin chain-assembly complex (LUBAC), that is essential to activate the canonical NFKB pathway and that limits caspase-8 and RIPK1 recruitment [4, 12]. High affinity IAP-antagonists are therefore attractive co-treatments to enhance programmed cell death in response to TNF $\alpha$ or TRAIL [13]. Besides cIAPs, also transforming growth factor $\beta$-activated kinase 1 (TAK1) has been implicated in regulating cell death susceptibility in this context. TAK1 contributes to pro-survival signaling by activating MAPK and NFkB pathways and additionally directly modulates apoptosis and necroptosis susceptibility by phosphorylating RIPK1 [14-16].

Previous work demonstrated that RIPK3-deficient melanoma cells understandably are necroptosis resistant [17]. Here, we therefore studied the cell death responsiveness and cell death modalities observed in melanoma cells that intrinsically express notable amounts of RIPK1, RIPK3 and MLKL. We likewise investigated the role of TAK1 in controlling cell death susceptibility and its potential as a prognostic marker for disease progression in melanoma.

\section{Materials and methods}

\section{Reagents and antibodies}

zVAD-fmk (Z-Val-Ala-Asp-fluoromethyl ketone) was purchased from Apexbio (MA, USA). The IAP antagonist compound A was generously provided by Tetralogics Corp. (Philadelphia, PA, USA). Necrostatin-1 was from Seleckchem (Houston, TX, USA). (5Z)-7-Oxozeaenol and MTT (3-(4,5-dimethylthiazol-2-yl)-2,5-diphenyltetrazolium bromide) were obtained from Sigma-Aldrich (St. Louis, USA). NG-25 was purchased from MedChem Express (New
Jersey, USA). Recombinant human TNF- $\alpha$ was purchased from R\&D Systems (Minneapolis, MN, USA). Fc-scTRAIL (TRAIL) was kindly provided by Prof. Roland Kontermann (University of Stuttgart, Germany). The following antibodies were used: rabbit polyclonal antibody RIPK3 (Cat No: IMG-5846A, IMGENEX, San Diego, Ca, USA), mouse monoclonal RIPK1 (Cat. No: R41220, Transduction Laboratories, San Diego, California), mouse monoclonal PARP (BD556494), rabbit monoclonal phospho-MLKL (ab187091), rabbit monoclonal phospho-RIPK1 (CST65746), rabbit monoclonal phospho-RIPK3 (ab209384), mouse monoclonal phospho-ERK1/2 (sc-7383), mouse monoclonal Caspase-8 (CST-9746), rabbit polyclonal Caspase-3 (CST-9662), rabbit monoclonal DNMT1 (CST5032), mouse monoclonal Actin (CST-3700), rabbit monoclonal TAK1 (CST-4505), rabbit monoclonal phospho-TAK1 (CST-4580), rabbit monoclonal NF-KB p65 (CST-8242), rabbit monoclonal phospho-NF-KB (CST3033), mouse monoclonal IкB $\alpha$ (CST-4814), rabbit monoclonal phospho-IкB $\alpha$ (CST-2859), mouse monoclonal phospho-p38 (CST-9216), rabbit polyclonal p38 (CST9212), mouse monoclonal ERK1/2 (CST-9107). Horseradish peroxidase (HRP)-conjugated goat anti-rabbit, goat anti-rat $\mathrm{IgG}$, and goat anti-mouse $\mathrm{IgG}$ were obtained from Dianova GmbH (Hamburg, Germany)

\section{Cell culture}

The following human melanoma cell lines were obtained either from the ATCC (WM115, SK-MEL 1, SK-MEL 5, WM793B, Malme-3M, MeWo, and SK-MEL 2; Manassas, VA, USA), the Wistar Institute (WM35, WM852, WM3211, WM1366, WM1346 and WM1791C; Philadelphia, PA, USA), the Deutsche Sammlung von Mikroorganismen und Zellkulturen GmbH (MEL-JUSO; Brunswick, Germany), Rockland Immunochemicals Inc (WM852 and WM3248; Limerick, PA, USA), Memorial Sloan-Kettering Cancer Center (WM1346 and SK-MEL 147; New York, USA). Eight cell lines carried activating BRAF mutations (WM35, WM793B, WM852, WM3248, WM115, SK-MEL 1, SK-MEL 5, and Malme-3M), five cell lines NRAS mutations (WM1366, WM1346, SK-MEL 147, SK-MEL 2, MEL-JUSO), one cell line a CDK4 mutation (WM1791C), one cell line carried a c-KIT mutation (WM3211), and one cell line was BRAF/NRAS/c-KIT/CDK4 wildtype (MeWo). Cells were cultured in Roswell Park Memorial Institute medium (RPMI) with $10 \%$ fetal calf serum, at $5 \% \mathrm{CO}_{2}$ at $37^{\circ} \mathrm{C}$. HaCaT keratinocytes were kindly provided by Petra Boukamp (DKFZ, Heidelberg, Germany) and cultured in RPMI medium as described above. Cell lines used in this study were tested regularly to be free of mycoplasm and were authenticated through STR profiles. 


\section{siRNAs, plasmids, and transfections}

The following siRNA duplexes were used: FlexiTube siRNA for caspase-8 (\#Hs_CASP8_11, Qiagen, Germany), for RIPK1 (\#Hs_RIPK1_5, Qiagen, Germany), and the corresponding control siRNA (AllStars Negative Control siRNA 1027281, Qiagen, Germany). siRNA (10 nM) and Lipofectamine RNAiMAX (Invitrogen, CA, USA) were diluted in Opti-MEM medium and incubated for $5 \mathrm{~min}$ at room temperature prior to addition to cells. Procedures followed the manufacturer's recommendations. Plasmids pCMV-TAK1-WT, pCMV-TAK1-K63W and empty vector control were kindly provided by Prof. Jun Ninomiya-Tsuji, NC State University, Raleigh, USA and described previously [18]. Cells were transfected by using ViaFect ${ }^{\mathrm{TM}}$ transfection reagent (Cat no: E4981; Promega Corporation, Madison, USA) according to the manufacturer's instructions.

\section{Generation of TAK1 deficient cells}

TAK1 deficient WM1791C cells were generated by the CRISPR/Cas9 approach. Cells were transfected with empty vector pSpCas9(BB)-2A-GFP-PX458 or a vector targeting TAK1 (Cat no: 48138, Addgene, Cat no: sc-400364, Santa Cruz Biotechnology). Two days after transfection, GFP positive clones were isolated by cell sorting (BD FACSAria $^{\mathrm{TM}}$ III, BD Biosciences) and plated as single clones in 96-well plates. Clones were cultured for 2-3 weeks and analysed for successful TAK1 knockout by Western blotting.

\section{MTT assay}

For viability assays, $0.5-2 \times 10^{4}$ cells were seeded per well of a 96 well plate and incubated overnight at $37^{\circ} \mathrm{C}$. Prior to experimental endpoints, cells were incubated with MTT at $5 \mathrm{mg} / \mathrm{ml}$ for $3-4 \mathrm{~h}$ and kept maintained in cell culture incubators. Medium was then aspirated, $100 \mu \mathrm{l}$ methanol were added, followed by $30 \mathrm{~min}$ incubation. The optical density of each well was measured with a TECAN multi reader (Infinite 200 PRO series, Männedorf, Switzerland) at $570 \mathrm{~nm}(\mathrm{OD} 570 \mathrm{~nm})$. Cellular viability was calculated using untreated controls as reference samples.

\section{Cell death assay}

Cells were collected and stained with propidium iodide (2 $\mu \mathrm{g} / \mathrm{ml}$ ) for $15 \mathrm{~min}$ in the dark at room temperature. Flow cytometric measurements were performed using a MACSQuant Analyzer 10 (Miltenyi Biotech) and data were analysed using Flowing Software (Turku Centre for Biotechnology, Finland).

\section{Western blotting}

Cells were washed with ice-cold PBS and lysed for $1 \mathrm{~h}$ on ice in cell lysis buffer (30 mM TRIS-HCl (pH 7.5), $120 \mathrm{mM}$ $\mathrm{NaCl}, 10 \%$ Glycerol, $1 \%$ Triton X, 1X Complete Protease Inhibitor (Roche, Mannheim, Germany). Cellular debris was removed by centrifugation at $20,000 \times g$ for $20 \mathrm{~min}$. Protein concentrations were quantified by Bradford assay. Equal amounts of proteins were supplemented with $5 \times$ Laemmli sample buffer and heated to $95{ }^{\circ} \mathrm{C}$ for $5 \mathrm{~min}$. Proteins were separated on Nu-Page 4-12\% Bis-Tris gels (Invitrogen, USA) and transferred to nitrocellulose membranes using an iBlot 2 gel transfer device (Invitrogen, Rockford, USA). After $1 \mathrm{~h}$ blocking with $5 \%$ bovine serum albumin (BSA) in Tris Buffered Saline with Tween-20 (TBST), the membranes were incubated with primary antibodies (diluted in 5\% BSA) overnight. After washing with TBST, the blots were incubated with horseradish peroxidase (HRP)-conjugated isotype-specific anti-mouse and/or anti-rabbit secondary antibodies. After further washing, chemiluminescence signals were detected on film or using an Amersham ${ }^{\mathrm{TM}}$ imager 600 system (Amersham Bioscience Europe, Freiburg, Germany).

\section{XMAP (luminex) assays}

Cells were cultured in 96-well plates, grown to near confluence and treated as indicated. Supernatants were transferred to separate 96-well plates and cells were lysed with ProATonce lysis buffer (ProtATonce, Athens, Greece) by freezing/thawing. Cellular debris was removed by centrifugation at $2700 \times g$ for $20 \mathrm{~min}$ and the protein concentration of the lysates was adjusted to $250 \mu \mathrm{g} / \mu \mathrm{l}$ using a BCA assay kit (Pierce ${ }^{\mathrm{TM}}$ ). xMAP bead-based ELISA type assays were performed on a Luminex FLEXMAP $3 \mathrm{D}^{\circledR}$ platform (Luminex, Austin, TX), using a custom-developed phosphoprotein 3-plex panel (ProtATonce, Athens, Greece): Mitogen-activated protein kinase-3 (ERK1/ MAPK3)-T202/Y204, Transcription factor AP-1 (c-JUN) - S63 and Transcription factor p65 (p65/Nf- $\mathrm{kB}$ ) - S536. For cytokine measurements, the protein concentrations of supernatants were adjusted to $100 \mu \mathrm{g} / \mu \mathrm{l}$ and cytokines were measured using a custom-developed 4-plex panel (ProtATonce, Athens, Greece): Interleukin-8 (IL8), Intercellular adhesion molecule (ICAM1), Follistatin (FST), chemokine (C-C motif) ligand 5 (CCL5) and Chemokine (C-X-C motif) ligand 1 (CCXL1).

\section{Survival analysis}

For analysis of primary melanoma data stored in the cancer genome atlas (TCGA), normalized mRNA expression data (Upper Quartile normalized Fragments per Kilobase of 
transcript per Million mapped read, $\log 2(\mathrm{FPKM}-\mathrm{UQ}+1)$ ) generated by the Genomic Data Commons (GDC-NIH) were downloaded from the UCSC-XENA browser (UCSC Xena. Available at: https://xena.ucsc.edu/. (Accessed: 24 October 2018). The "disease free survival from sample procurement" was calculated as the difference between the "disease free survival from initial diagnosis" and "sample procurement from initial diagnosis", downloaded from cBioPortal [19] and the GDC data portal (https://portal.gdc. cancer.gov/. (Accessed: 25 October 2018)), respectively. If no recurrence or progression events were reported, the date of last follow up or death were considered as final time points. An optimized chi-squared based separator was determined to divide patients with high and low MAP $3 K 7$ (TAK1) mRNA expression, and the two groups were compared by log-rank test. The optimal separator resulted in the highest chi-squared value with the function survdiff of the library survival in R (version 3.4.0), and the difference in mean survival time at different cut-off points was calculated and plotted with the tool Cutoff Finder [20]. Kaplan-Meier survival curves were generated and compared using GraphPad Prism 4.

\section{Statistical analysis}

Statistical analysis was performed using GraphPad Prism 7. If not stated otherwise, the mean values of three independently conducted experiments are plotted, where bars sh7ow the standard error of mean. Data were analyzed for statistically significant differences using One-way ANOVA and Bonferroni correction for multiple testing. A confidence level of $95 \%$ was used for all analyses $(\alpha$-value $=0.05)$. Results were summarized as $* * * * p<0.0001 ; * * * p<0.001$; ${ }^{* *} p<0.01 ; * p<0.05 ;$ n.s. $=$ not significant.

\section{Results}

\section{Melanoma cells expressing RIPK1, RIPK3 and MLKL are necroptosis resistant}

Cancer cells frequently downregulate key necroptosis actors, in particular RIPK3 [17, 21, 22]. To study necroptosis competency, we therefore initially assessed expression heterogeneity of the essential RIPK1-RIPK3-MLKL necroptosis axis in melanoma cell lines. Across a panel of 16 cell lines, including cell lines harboring activating BRAF and NRAS mutations (see materials and methods), we noted comparable RIPK1 expression whereas RIPK3 and MLKL amounts differed considerably (Fig. 1a). RIPK3 was absent in many cell lines but frequently also clearly detectable, up to amounts similar to those observed in whole cell protein extracts from necroptosis competent $\mathrm{HaCat}$ keratinocytes
[17]. With the exception of SK-MEL-2 and MeWo cells, all other cell lines expressed clearly detectable but differing amounts of MLKL (Fig. 1a). All cell lines expressed comparable amounts of procaspase- 8 and cFLIP, as regulators of apoptosis signal transduction and, with exception of cIAP2, also comparable amounts of XIAP and cIAP1, as negative regulators of both apoptosis and necroptosis signaling [3] (Fig. 1b). To study necroptosis competency, we selected Malme-3M, MelJuso, WM1791C and WM3211 cells for subsequent experiments, since these cells expressed RIPK3 and MLKL, albeit in differing amounts.

TNF-receptor pathways can induce necroptosis, in particular when combining TNF receptor ligands with IAP antagonists and, to suppress apoptosis signaling, caspase inhibitors such as zVAD-fmk [23]. With the exception of moderately responsive MelJuso cells, all cell lines were largely resistant to TNF $\alpha$ or a multivalent TRAIL variant [24] (Fig. 1c). IAP antagonist Compound A reduced viability in Malme-3M cells, but only at very high concentrations (Fig. 1c). The combination of TNF $\alpha$ and Compound A most prominently reduced cell viability in WM3211 and Malme-3M cells, and the combination with caspase inhibitor zVAD-fmk (zVAD) failed to reduce viability further, indicating that canonical necroptosis cannot be induced (Fig. 1d). Correspondingly, RIPK1 inhibitor necrostatin-1 (nec-1) did not notably prevent viability loss in WM3211 and Malme-3M cells. WM1791C and MELJUSO cells likewise failed to die by necroptosis (Fig. 1d). Similar results were obtained in TRAIL-based treatment scenarios, where combination treatment with Compound A prominently reduced cell viability, and with zVAD but not nec-1 offering protection (Fig. 1d), indicating that cell death proceeds by apoptosis rather than necroptosis. Indeed, depleting caspase-8 expression in melanoma cells was sufficient to prevent cell death (Supplementary Fig. 1). In contrast, nec-1 or the combination of zVAD and nec-1 rescued necroptosis competent HT29 cells from TNF $\alpha /$ Compound A or TRAIL/Compound A treatment (Fig. 1e). Taken together, these data therefore demonstrate that melanoma cell lines with evident expression of the key actors of the canonical necroptosis cascade, namely RIPK1, RIPK3, MLKL, are incapable of dying by necroptosis.

\section{Elevating intrinsic RIP3 expression fails to establish necroptosis competency in melanoma}

It was previously demonstrated that DNA methyltransferase activity might silence RIPK3 expression [22, 25]. We therefore reasoned that treatment with 5-aza-2'-deoxycytidine (5-AD), a demethylating agent that induces the degradation of DNA (cytosine-5)-methyltransferase 1 (DNMT1) [26], could increase intrinsic RIPK3 expression and therefore necroptosis competency in melanoma cells. Indeed, 5-AD treatment 
A
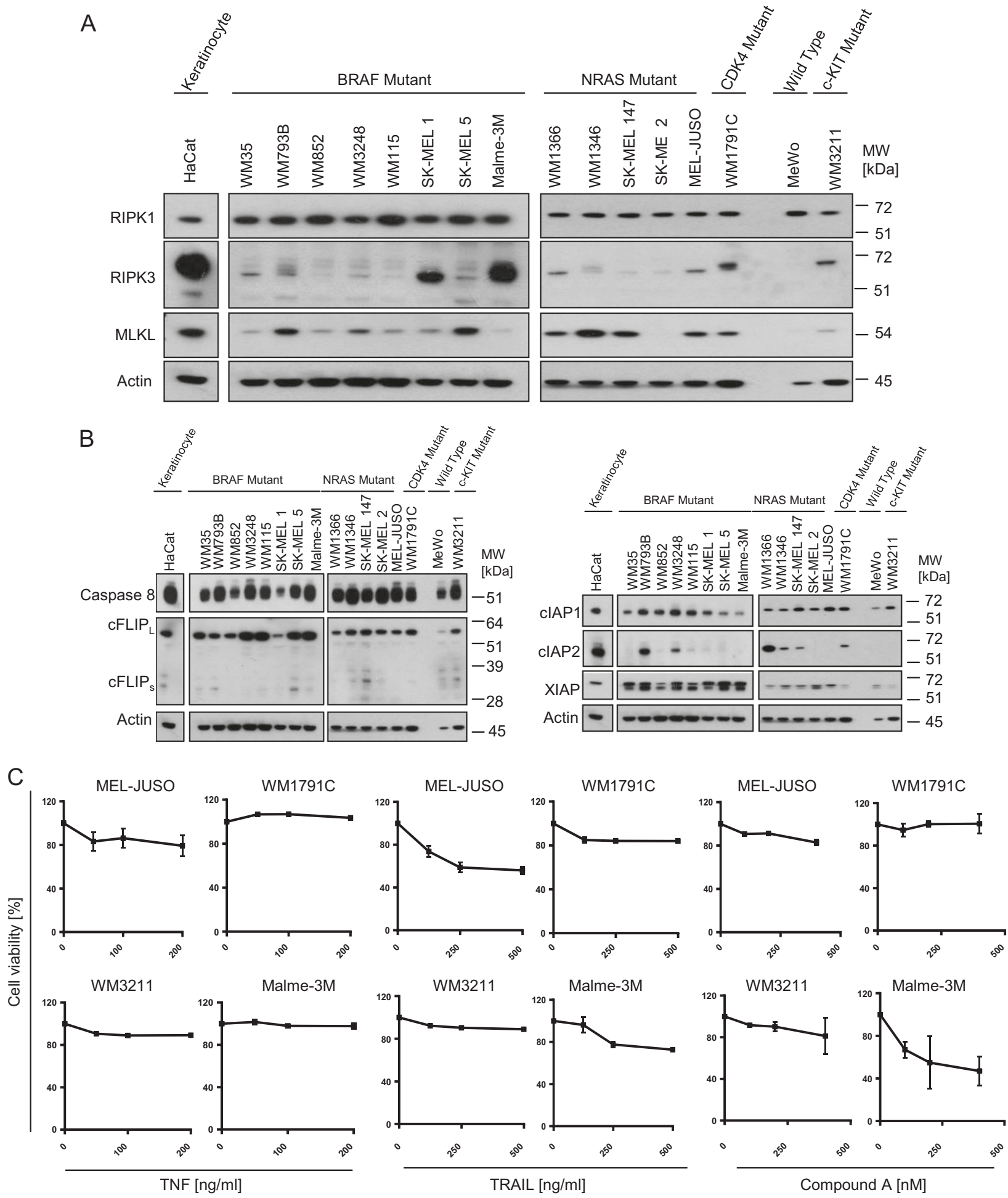

Fig. 1 Melanoma cells expressing RIPK1, RIPK3 and MLKL are necroptosis resistant. $\mathbf{a}, \mathbf{b}$ Basal expression of key cell death regulatory proteins in melanoma cell lines was determined by immunoblotting. Necroptosis-competent HaCat keratinocytes served for comparison. Actin served as loading control. c Cell viability of MEL-JUSO, WM1791C, WM3211, and Malme-3M cell lines in response to TNF $\alpha$, TRAIL or IAP antagonist Compound A, as determined by MTT assays. Cells were treated for $24 \mathrm{~h}$. Data show mean \pm S.E.M. from $n$ $=3$ independent experiments. d, e Cell viability in response to combination treatment of TNF $\alpha$ or TRAIL with IAP antagonist Compound $\mathrm{A}$, as determined by MTT assays. Cells were treated for $48 \mathrm{~h}$. zVADfmk and Necrostatin 1 were added 30 min prior to treatment. HT-29 cells served as necroptosis competent control. Data show mean \pm S.E. M. from $n=3$ independent experiments 


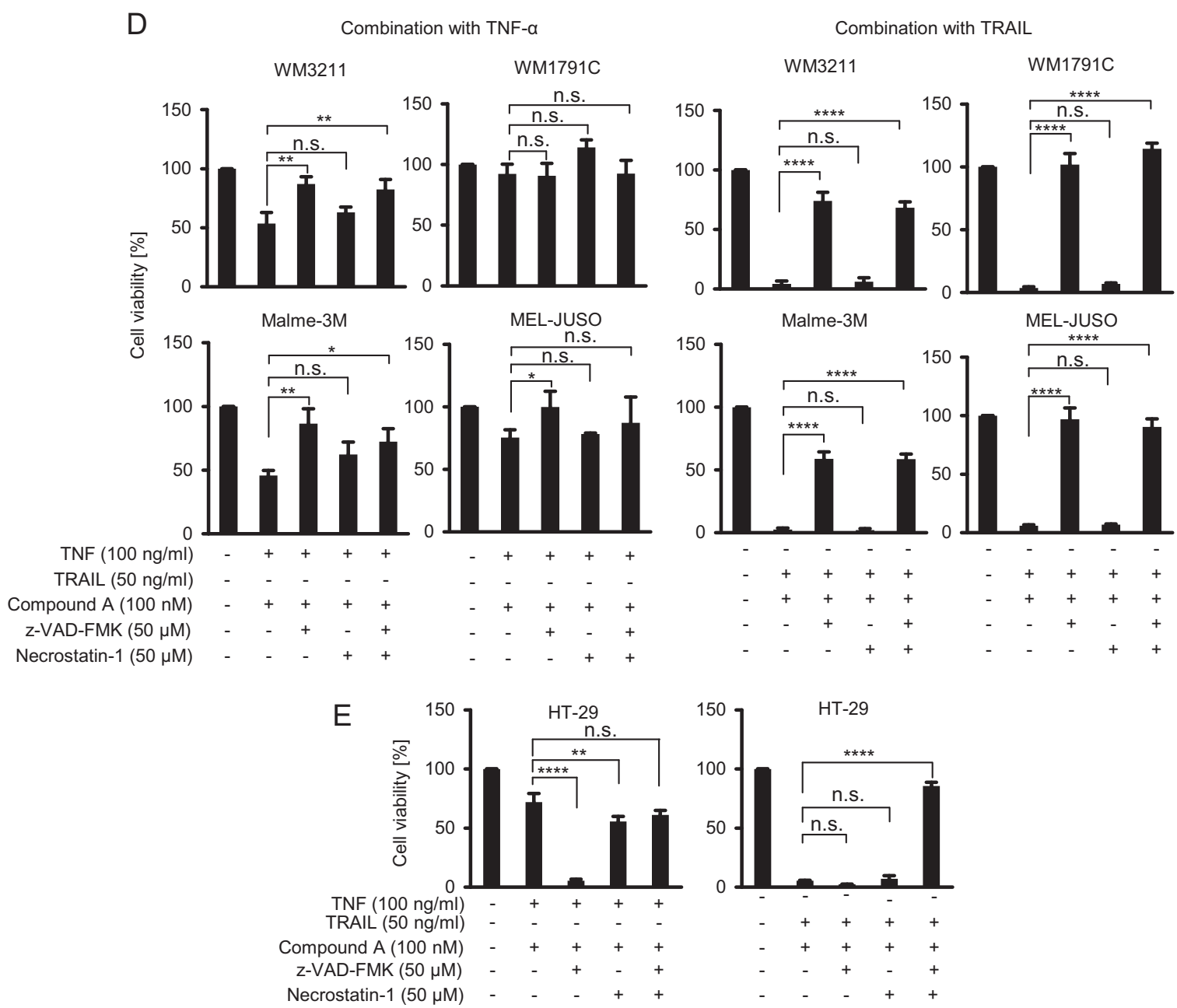

Fig. 1 continued

notably enhanced RIPK3 expression in all cell lines, whereas RIPK1 expression remained unaffected (Fig. 2a). In MALME-3M and MEL-JUSO cells, also MLKL expression increased following 5-AD treatment (Fig. 2a). As expected, DNMT1 expression was lost in cells exposed to 5-AD (Fig. 2b). Importantly, RIPK3 and MLKL protein amounts remained elevated for several days after 5-AD withdrawal (Fig. 2c), indicating that 5-AD pre-treatment might pose an attractive strategy for priming cells towards RIPK3/MLKLdependent necroptosis. However, despite substantially elevated RIPK3 expression, no substantial induction of necroptosis could be observed (Fig. 2d), indicating that signal transduction towards necroptosis is likely inhibited upstream of RIPK3/MLKL signaling.

\section{TAK1 inhibitor (5Z)-7-0xozeaenol establishes RIPK1- dependent apoptosis but not necroptosis competency in melanoma}

Besides activating NFKB and MAPK signaling pathways, the transforming growth factor- $\beta$-activated kinase 1 (TAK1) has been implicated in suppressing RIPK1 activity and thereby to suppress necroptosis signaling [16]. We therefore reasoned that antagonizing TAK1 activity, using the high affinity inhibitor (5Z)-7-oxozeaenol (Oxo) [27, 28], might restore necroptosis competency in malignant melanoma. As expected, Oxo suppressed NFкB and MAPK signaling, as demonstrated by loss of phosphorylated p65 and IкB $\alpha$ (Fig. 3a) and by reductions in p38 and ERK1/2 phosphorylation (Fig. 3b). Oxo did not affect the expression of RIPK1, RIPK3 or MLKL (Fig. 3c).

As a single drug or in combination with Compound A, Oxo was only modestly effective in reducing melanoma cell viability, whereas the triple combination with $\mathrm{TNF} \alpha$ strongly reduced cellular viability in all cell lines tested (Fig. 3d). A similar effect was observed when using an alternative TAK-1 inhibitor (NG-25) (Fig. 3e). Viability loss in WM3211 was completely caspase dependent, whereas in WM1791C cells, and to a lesser extent in Malme-3M cells, RIPK1 inhibition by nec-1 prevented viability loss (Fig. 3d). In MEL-JUSO cells, nec-1 reduced viability loss only in combination with caspase-inhibitor 

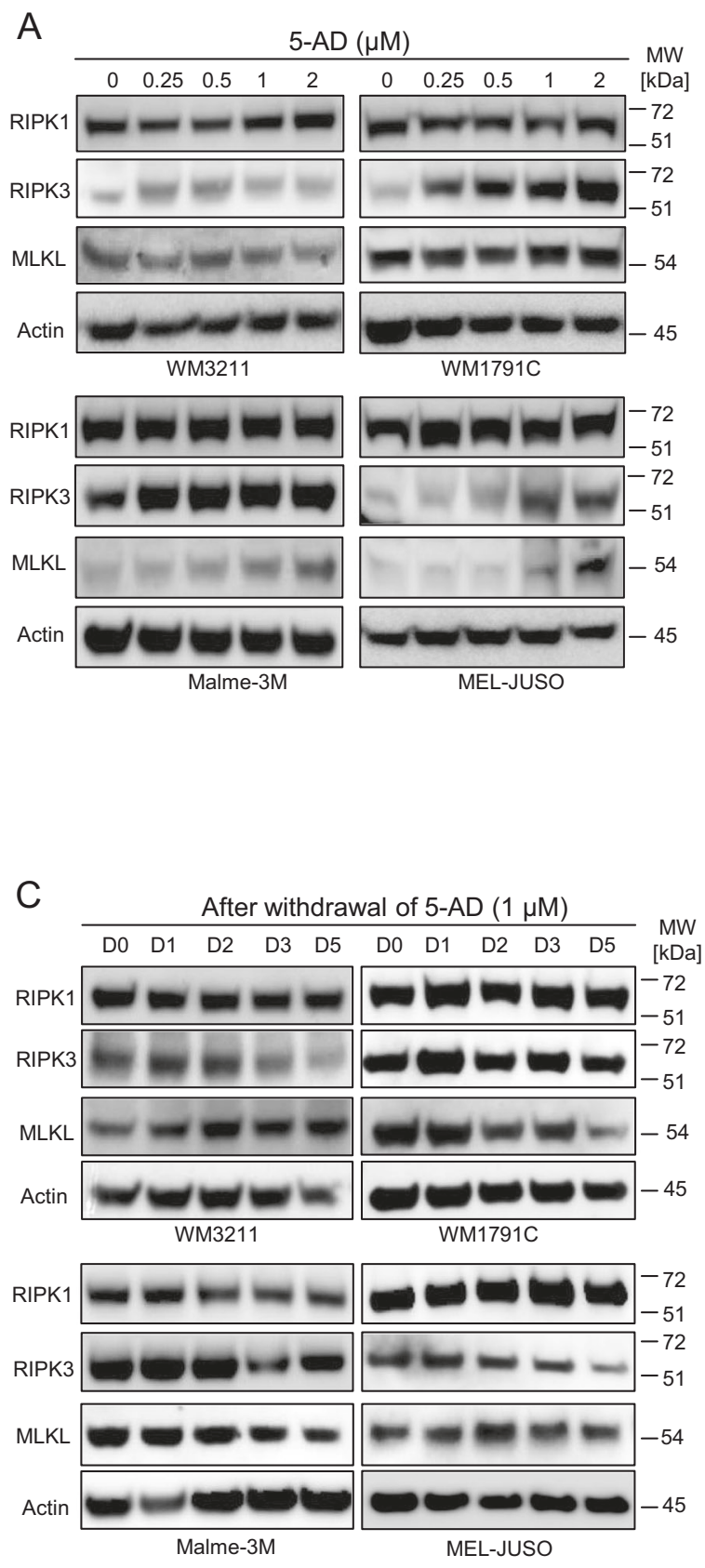

Fig. 2 Elevating intrinsic RIP3 expression fails to establish necroptosis competency in melanoma. a Melanoma cells were treated with 5-AD for 4 days and whole cell extracts were probed for RIPK3, RIPK1, MLKL expression amounts by immunoblotting. Actin served as loading control. b DNMT1 expression was determined following 2 days of 5-AD treatment by immunoblotting. Actin served as loading

zVAD (Fig. 3d). In contrast to necroptosis competent HT29 cells (Fig. 3f), Oxo therefore established conditions of caspase and RIPK1-dependent cell death in $\mathrm{TNF} \alpha / \mathrm{Com}$ pound A-treated cells, as seen prominently in WM1791C cells. Interestingly, cell death remained completely
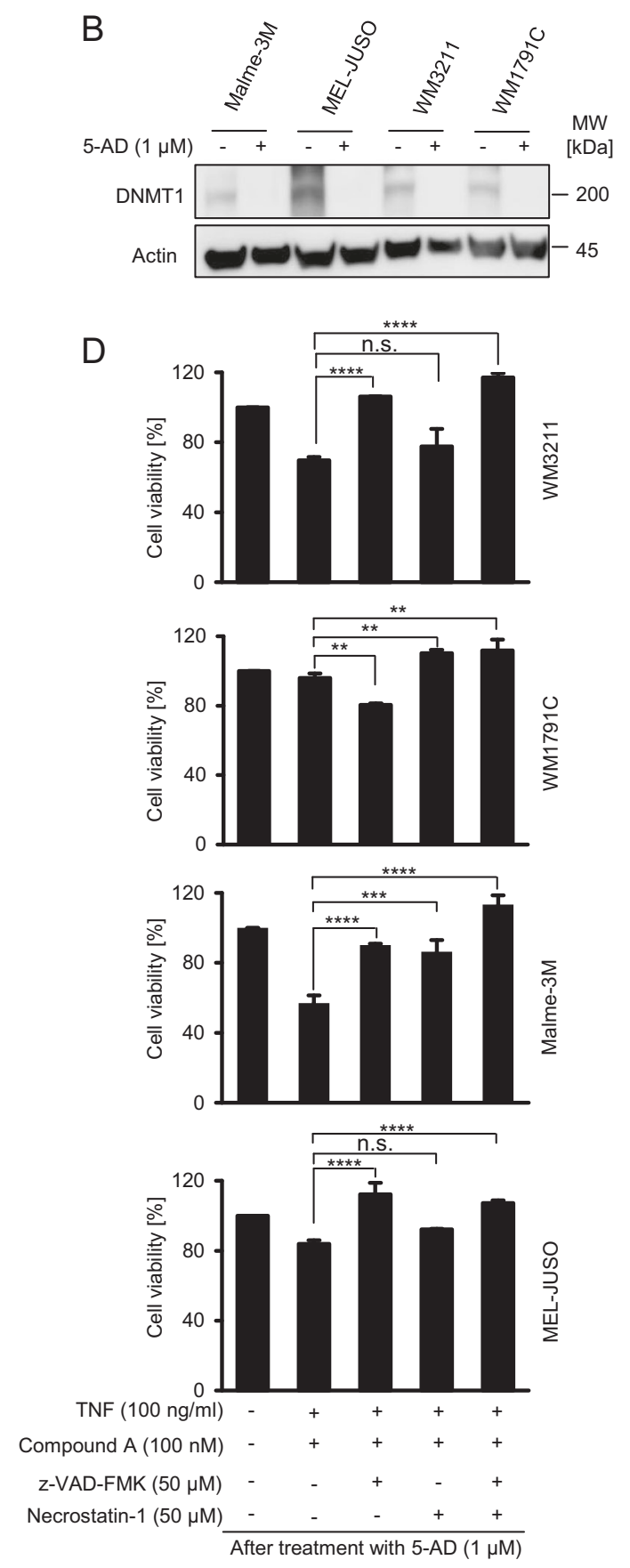

control. c Elevated RIPK3 expression persists after 5-AD withdrawal. As in (a), protein amounts were probed in melanoma cell populations up to 5 days after 5-AD washout. $\mathbf{d}$ Cell viability of melanoma cells in which intrinsic RIPK3 expression was elevated by 5-AD pre-treatment. Viability was determined by MTT assays. Data show mean \pm S.E.M. from $n=3$ independent experiments

independent of RIPK1 in TRAIL-based treatment scenarios (Supplementary Fig. 2).

In addition, Oxo notably suppressed TNF $\alpha$ and/or Compound A-induced pro-survival NFKB signaling, as demonstrated by reduced amounts of phosphorylated $\mathrm{p} 65$ 
A

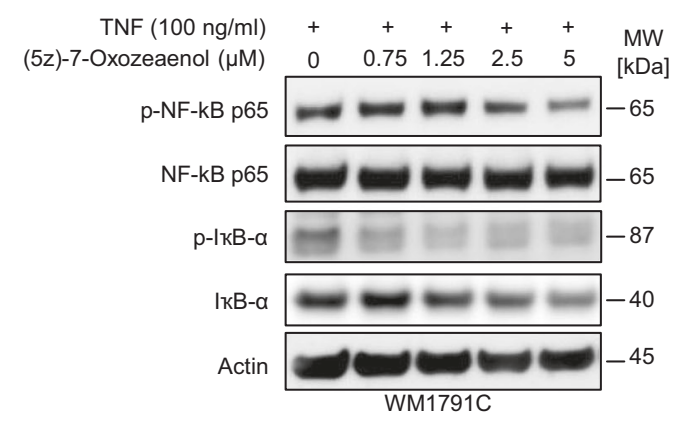

C

E
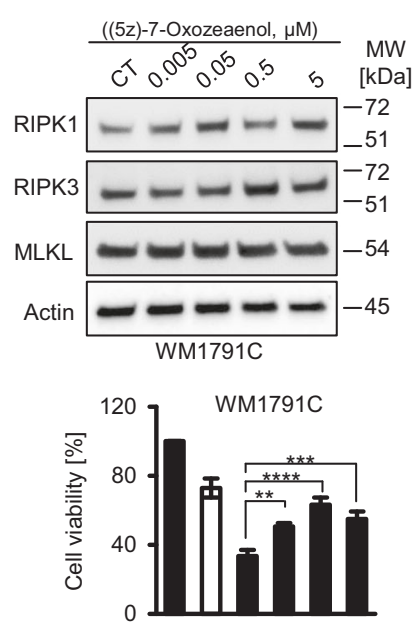

TNF (100 ng/ml)

NG-25 $(5 \mu \mathrm{M}) \quad-+++++$

Compound $\mathrm{A}(100 \mathrm{nM}) \quad-\quad+\quad+\quad++$

z-VAD-FMK $(50 \mu \mathrm{M}) \quad-\quad-\quad+\quad-+$

Necrostatin-1 $(50 \mu \mathrm{M}) \quad-\quad-\quad-\quad++$

$\mathrm{F}$

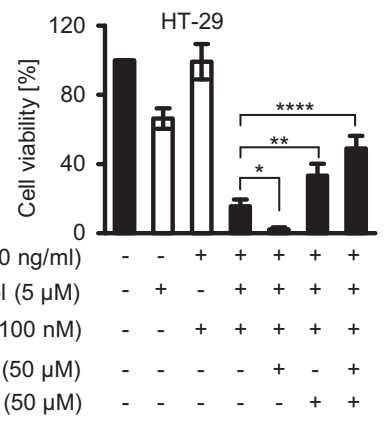

Fig. 3 (5Z)-7-Oxozeaenol establishes conditions of caspase- and RIPK1-dependent cell death in TNF $\alpha /$ Compound A-treated cells. a, b WM1791C cells were pre-treated for 30 min with 5z-7-oxozeaenol, followed by $30 \mathrm{~min}$ of TNF- $\alpha$ stimulation. Whole cell extracts were studied by immunoblotting. Actin served as loading control. c WM1791C cells were treated for $2 \mathrm{~h}$ and whole cell extracts were probed for RIPK1, RIPK3, and MLKL expression amounts by
B $\operatorname{TNF}(100 \mathrm{ng} / \mathrm{ml})+++++\mathrm{MW}$

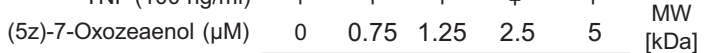
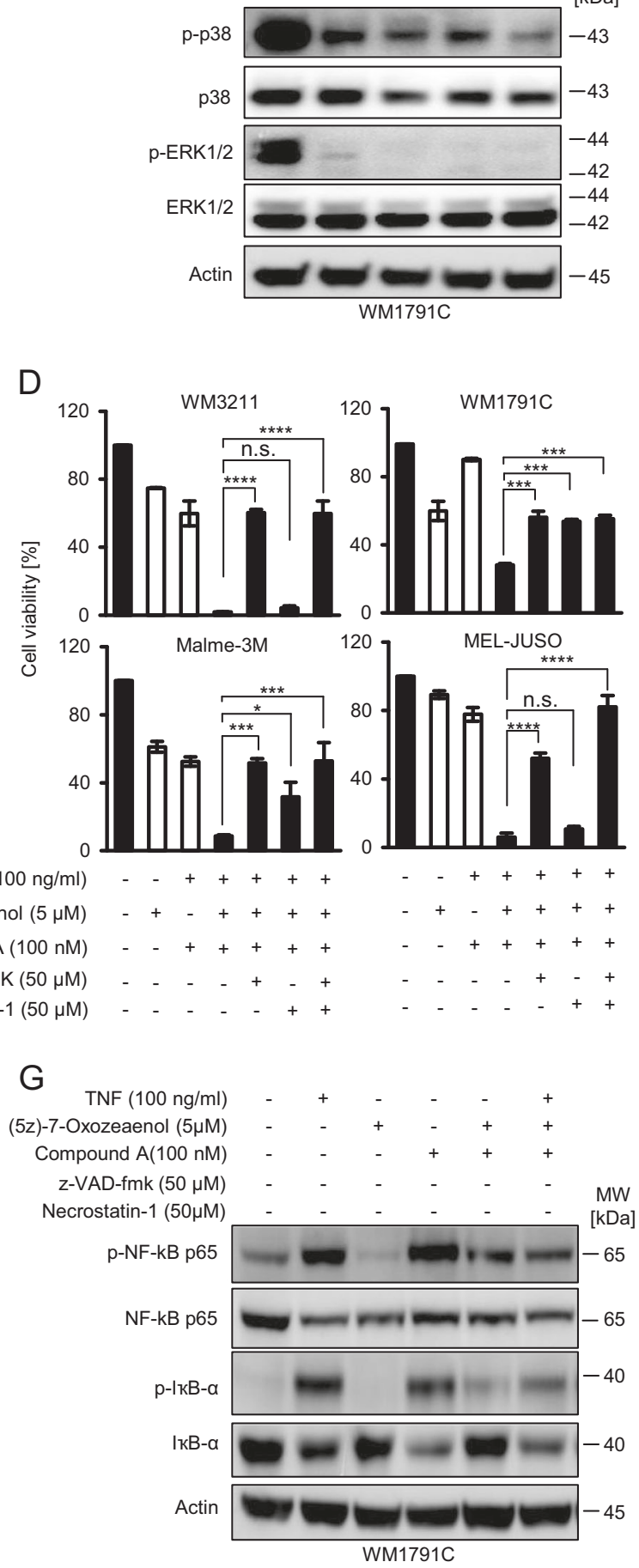

immunoblotting. Actin served as loading control. d-f Cell viability of melanoma cells or HT29 cells treated as indicated. Viability was determined by MTT assays following $24 \mathrm{~h}$ of treatment. Data show mean \pm S.E.M. from $n=3$ independent experiments. g WM 1791C cells were treated as indicated. Whole cell extracts were analyzed $2 \mathrm{~h}$ after stimulation with TNF $\alpha$. Actin served as loading control 
and $\mathrm{I} \kappa \mathrm{B} \alpha$ in Oxo-combination treatments (Fig. 3g). Luminex-based phosphoprotein measurements provided similar results, with Oxo preventing basal or TNF $\alpha / C o m-$ pound A-induced phosphorylation of p65, Jun kinase and MAP kinase 3 (MAPK3) (Supplementary Fig. 3A). Oxo treatments also reduced basal or $\mathrm{TNF} \alpha /$ Compound Ainduced secretion of cytokines and soluble factors that were previously linked to melanoma disease progression and escape from immune surveillance, such as interleukin-8 (IL8) [29, 30], chemokine (C-X-C motif) ligand 1 (CXCL1) [31, 32], chemokine (C-C motif) ligand 5 (CCL5) [33], follistatin (FST) [34], and shedded intercellular adhesion molecule 1 (ICAM-1) [35] (Supplementary Fig. 3b).

Overall, these data therefore indicate that TAK-1 inhibitor Oxo enhances cell death susceptibility of melanoma cells exposed to $\mathrm{TNF} \alpha / \mathrm{Compound} \mathrm{A}$. To more specifically test for RIPK1 or caspase-8 dependency in this treatment scenario, we depleted RIPK1 and caspase-8 expression in WM1791C cells. In line with our previous results, viability loss required the presence of RIPK1 and caspase-8 (Fig. 4a). Processing of caspase- 8 and 3 as well as PARP cleavage was prominently detectable after treatment with TNF $\alpha$, Compound A and Oxo, with both zVAD and nec-1 suppressing this apoptotic cascade (Fig. 4b). Cell morphologies and cell death measurements by PI staining further supported that cell death was executed primarily by RIPK1 dependent apoptosis (Fig. 4c, d). Taken together, Oxo therefore appears to enhance the competency of melanoma cells to execute RIPK1 dependent apoptosis, but not necroptosis.

\section{Necroptosis remains blocked upon combination of 5-AD pre-treatment and addition of (5Z)-7- oxozeaenol}

Since Oxo treatment established conditions of RIPK1dependent apoptosis in melanoma cells, we next tested if absence of necroptosis was attributable to the low expression of RIPK3 in melanoma. We therefore used melanoma cells pre-treated with 5-AD (see Fig. 2) to test if combination treatments with $\mathrm{TNF} \alpha / \mathrm{Compound} \mathrm{A} / \mathrm{Oxo}$ were now capable of inducing necroptotic cell death. As seen in Fig. 5a, none of the cell lines studied died by necroptotic cell death. However, the dependency on RIPK1 activity increased in WM3211 and WM1791C cells, as seen when combining zVAD and nec-1 (Fig. 5a), possibly due to elevated amounts of RIPK3 supporting RIPK1-dependent apoptosis in this setting [36]. To study how far necroptosis signaling is transduced through the RIPK1, RIPK3, MLKL cascade, we studied the phosphorylation status of these proteins. While the conventional necroptosis-inducing treatment of $\mathrm{TNF} \alpha / \mathrm{Compound} \mathrm{A} / \mathrm{zVAD}$ resulted in strong auto-phosphorylation of RIPK1 at Ser166 and in phosphorylation of RIPK3 (Ser227) and MLKL (Ser358) in HT-29 cells, not even RIPK1 was phosphorylated in WM1791C cells (Fig. 5b). This finding indicates early termination of necroptosis signaling in the latter cell line. In WM1791C cells pre-treated with 5-AD and exposed to treatment combinations as in Fig. 5a, RIPK1 autophosphorylation was observed in combination with the triple treatment of $\mathrm{TNF} \alpha /$ Compound $\mathrm{A} / \mathrm{Oxo}$, but only when caspase activation was prevented (Fig. 5c). Caspase activity therefore seems to prevent RIPK1 oligomerisation, as needed for auto-phosphorylation and as seen in necroptosis competent HT-29 cells, and caspase inhibition was insufficient for signaling to proceed from RIPK1 towards RIPK3 and MLKL.

\section{TAK-1 inactivation is essential for RIPK1-dependent apoptosis in melanoma}

Even though Oxo is a high affinity inhibitor of TAK1, off target effects cannot be excluded based on its selectivity profile [28]. We therefore performed genetic studies to test the relevance of TAK1 in preventing RIPK1-dependent apoptosis in melanoma more specifically. First, we eliminated TAK1 expression by CRISPR/Cas9 targeting of the $M A P 3 K 7$ gene and studied viability loss after treatment with TNFo/Compound A (Fig. 6a, b). WM1791C cells lacking TAK1, but not parental control cells, were substantially sensitized to treatment with $\mathrm{TNF} \alpha / \mathrm{Compound} \mathrm{A}$, and cell death could be completely prevented by zVAD or nec-1 (Fig. 6b). Cell morphologies and propidium iodide uptake returned similar results, indicating apoptosis induction specifically for the TNF $\alpha /$ Compound A combination treatment in cells lacking TAK1 (Fig. 6c). We next restored TAK1 expression by introducing either wildtype or kinase dead TAK1 (Fig. 6d). Only wildtype TAK1 but not inactive TAK1 protected against the combination of TNF $\alpha / C o m-$ pound A (Fig. 6e), demonstrating TAK1 and specifically its kinase activity to be a key suppressor of RIP1-dependent apoptosis in melanoma.

Since TNF $\alpha$ can be found within melanoma microenvironments [37], elevated expression of TAK1 in melanoma cells could contribute to preventing TNF $\alpha$-induced apoptotic signaling and additionally might promote migration and invasion through other signaling pathways, as was shown in breast and colon cancer [38]. We therefore studied how the expression of MAP $3 K 7$ relates to disease progression in melanoma in a cohort of $n=102$ patients diagnosed with primary melanoma for which dates of sample procurement and sufficient follow up information was available (Fig. 7a). The mean disease free survival between patients with low and high MAP $3 K 7$ expression in primary melanomas differed across a range of group separator thresholds (Fig. 7b), with significantly better prognosis for patients with low MAP3K7 expression (Fig. 7c). These results therefore extend our findings on the cell death-suppressing role of TAK1 in melanoma cell lines 
A

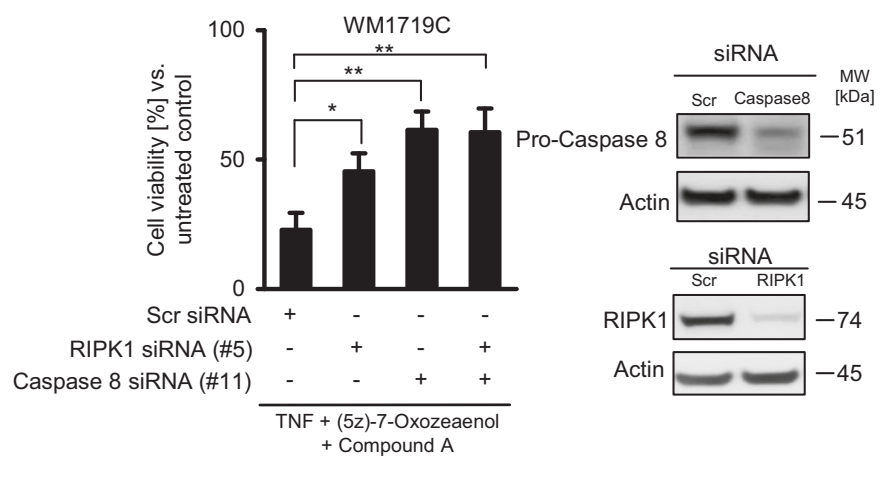

B

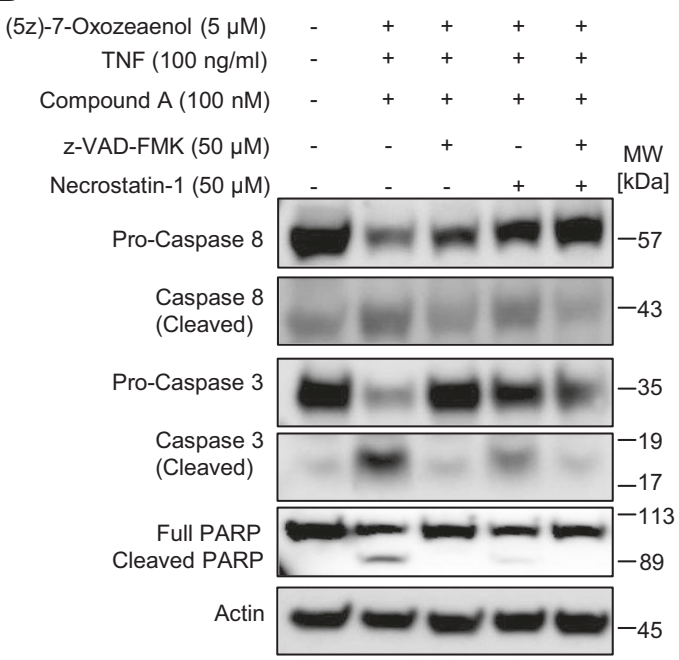

C

TNF $(100 \mathrm{ng} / \mathrm{ml})$

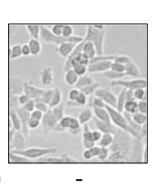

(5z)-7-Oxozeaenol $(5 \mu \mathrm{M})$

Compound A (100 nM)

z-VAD-FMK $(50 \mu \mathrm{M})$ Necrostatin-1 $(50 \mu \mathrm{M})$

D
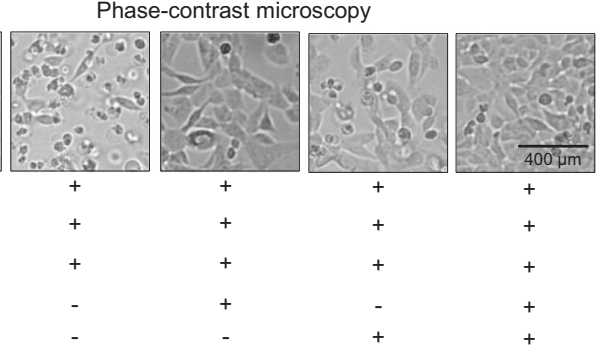

WM1791C

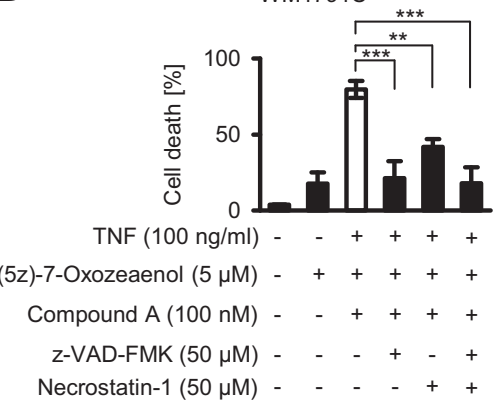

Fig. 4 Cell death sensitization by (5Z)-7-Oxozeaenol requires the presence of RIPK1 and caspase-8. a Cell viability of WM1791C cells in which RIPK1 and/or caspase-8 expression were depleted by siRNA transfection $(48 \mathrm{~h})$, following $24 \mathrm{~h}$ of treatment. Data show mean $\pm \mathrm{S}$. E.M. from $n=3$ independent experiments. Immunoblots demonstrate depletion efficiency. b WM1791C cells were treated as indicated and

towards delineating a role of TAK1 in melanoma pathophysiology and patient outcome.

\section{Discussion}

Here, we report that melanoma cells expressing the key players of necroptosis signal transduction fail to execute necroptotic cell death. However, signaling triggered by TNF whole cell extracts were studied by immunoblotting. Actin served as loading control. c Cells were treated for $24 \mathrm{~h}$ as indicated and observed by phase contrast microscopy. d Cell death quantification for WM1791C cells treated for $24 \mathrm{~h}$ as indicated. Cell death was determined by flow cytometric measurement of propidium iodide positive cells. Data show means \pm S.E.M. from $n=3$ independent experiments

receptor activation can be directed towards RIPK1dependent apoptosis by inhibition of TAK1. Indeed, low expression of TAK1 is associated with a more favorable prognosis for patients diagnosed with primary melanoma.

Whereas RIPK1 was expressed homogeneously across the panel of melanoma cell lines studied here, we noted considerable heterogeneity in RIPK3 and MLKL expression. While primary melanocytes and nevus cells express RIPK3 [17], RIPK3 expression appears to be lost during 


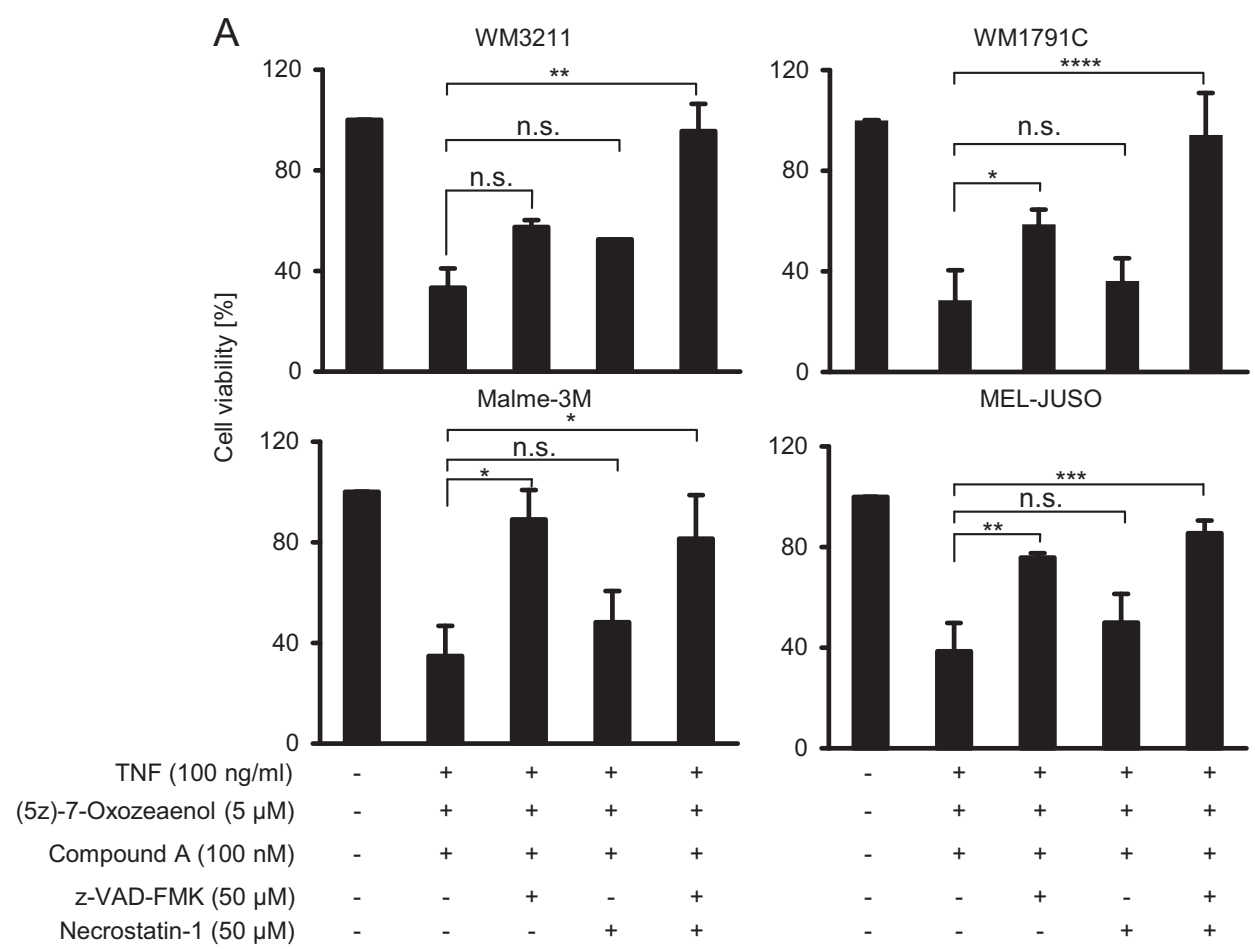

B

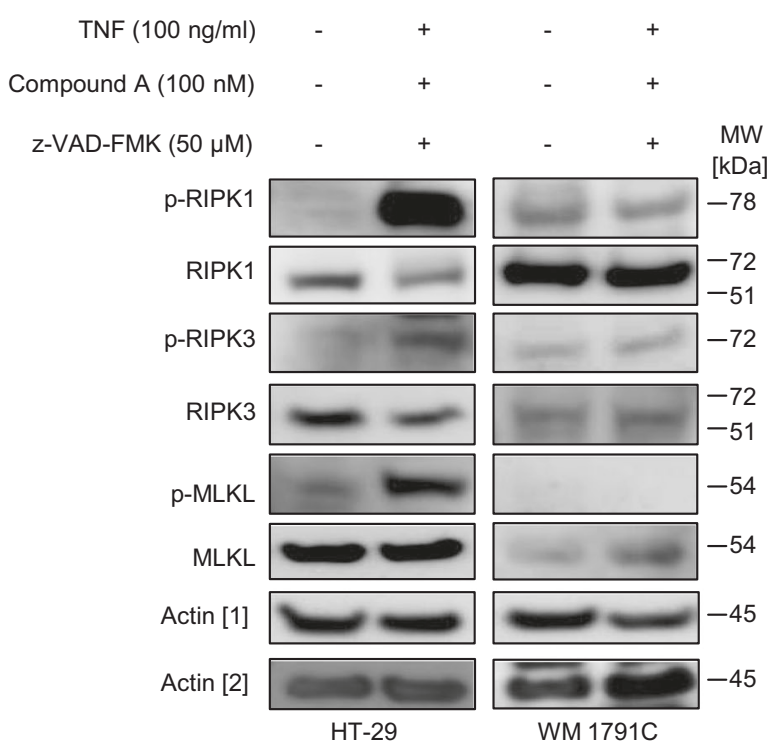

C

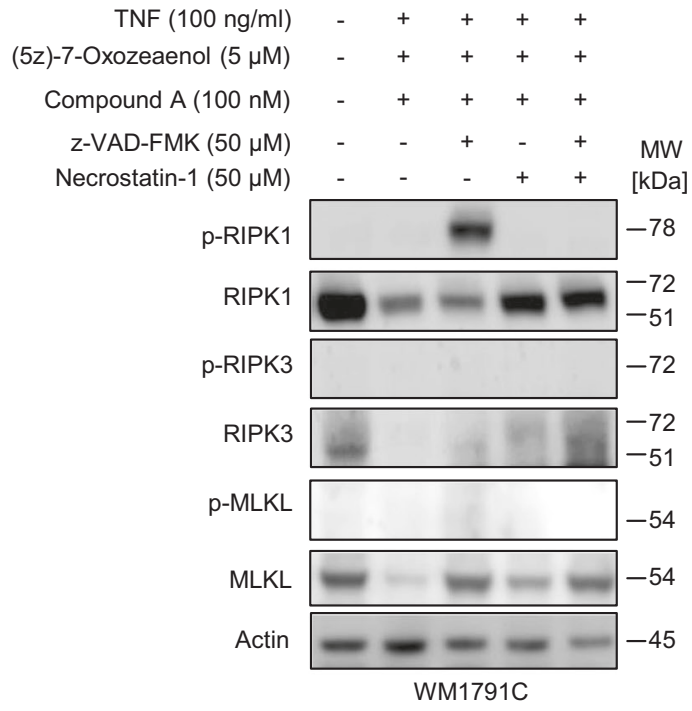

Fig. 5 Necroptosis remains blocked upon combination treatment with 5-AD and (5Z)-7-oxozeaenol. a Cell lines in which RIPK3 expression was restored by 5 -AD treatment $(1 \mu \mathrm{M} ; 4 \mathrm{~d})$ were subsequently treated as indicated. Cell viability was measured by MTT assays. Data show means \pm S.E.M. from $n=3$ independent experiments. b HT-29 and WM1791C cells were treated for $24 \mathrm{~h}$ as indicated and whole cell

melanomagenesis and is typically absent in most commonly used cell line models. Interestingly, the presence of BRAF and AXL oncogenes correlates with a loss in RIPK3 expression, as was recently shown in a large cell line panel extracts were probed for the amounts of phosphorylated or overall RIPK1, RIPK3 and MLKL. Actin served as loading control. c WM1791C cells were treated with 5 -AD for $4 \mathrm{~d}$ and afterwards treated for $24 \mathrm{~h}$, as indicated. Whole cell extracts were probed for the amounts of phosphorylated or overall RIPK1, RIPK3 and MLKL. Actin served as loading control

[21]. In our melanoma cell line panel, the majority of BRAF mutated cells lines expressed low or undetectable amounts of RIPK3, with the exception of SK-MEL-1 and Malme$3 \mathrm{M}$, both of which expressed very high amounts of RIPK3. 
A

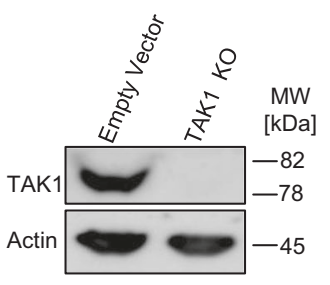

C

$$
\begin{array}{r}
\text { TNF }(100 \mathrm{ng} / \mathrm{ml}) \\
\text { Compound A }(100 \mathrm{nM}) \\
\text { z-VAD-FMK }(50 \mu \mathrm{M}) \\
\text { Necrostatin-1 }(50 \mu \mathrm{M})
\end{array}
$$

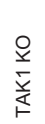

B
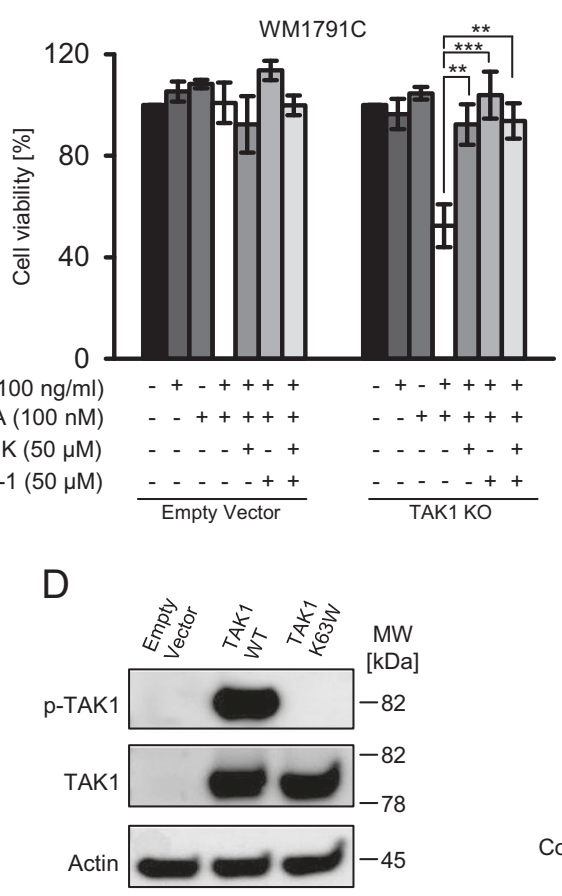

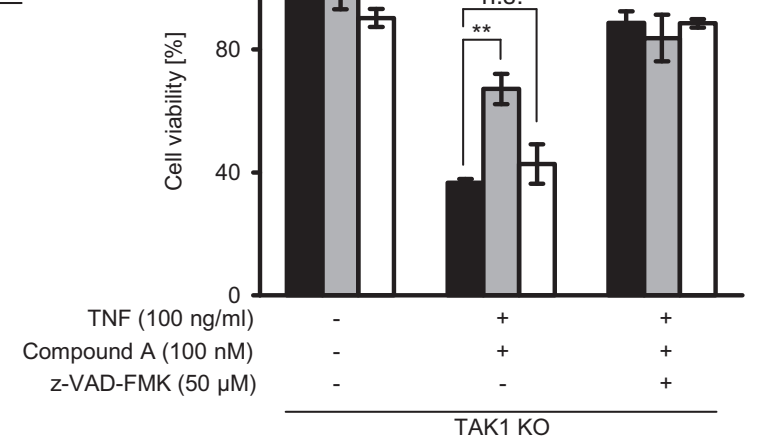

Fig. 6 TAK-1 inactivation is essential for RIPK1-dependent apoptosis in melanoma. a Confirmation of TAK1 knock out in WM1719C cells by immunoblotting. Actin served as loading control. b Control and TAK1-deficient WM1719C cells were treated for $24 \mathrm{~h}$ as indicated. Cell viability was determined by MTT assay. Data show means \pm S.E. M. from $n=3$ independent experiments. c Cells were treated for $24 \mathrm{~h}$ as indicated and observed by phase contrast microscopy. Cell death was subsequently determined by flow cytometric measurement of

How BRAF or AXL activity suppresses the expression of RIPK3 and how relevant and broadly applicable such a mechanism is in the melanoma setting, however, will need further investigation. We showed that silencing of RIPK3 expression in melanoma likely depends on DNA methylation, since 5-AD treatment and the associated degradation of DNMT1 correlated with the accumulation of RIPK3. Indeed, DNMT1 can methylate the RIPK3 promotor, thereby silencing RIPK3 expression and increasing necroptosis resistance of otherwise necroptosis competent propidium iodide positive cells. Data show means \pm S.E.M. from $n=3$ independent experiments. d Confirmation of TAK1 reconstitution (wild type or $\mathrm{K} 63 \mathrm{~W}$ ) by immunoblotting, following transient transfection with respective expression vectors. e Control and TAK1reconstituted cells were treated for $24 \mathrm{~h}$ as indicated. Cell viability was determined by MTT assay. Data show means \pm S.E.M. from $n=3$ independent experiments

cells such as mouse embryonic fibroblasts and L929 cells as well as human HT29 cells [25]. In reverse, RIPK3 promoter demethylation is required and sufficient to restore RIPK3 expression in various human cancer cell models [22]. However, melanoma cells so far were not studied in this context, neither were treatment conditions used that would unequivocally confirm that restoring RIPK3 expression is sufficient to enhance canonical necroptosis signal transduction. We demonstrate that increasing intrinsic RIPK3 expression by demethylating agent 5-AD is insufficient to 
A

\begin{tabular}{|c|c|c|}
\hline \multicolumn{3}{|c|}{ Patients demographics and clinical information } \\
\hline \multicolumn{3}{|l|}{ Age at diagnosis (years) } \\
\hline Mean & \multicolumn{2}{|c|}{64.78} \\
\hline Median & \multicolumn{2}{|c|}{65} \\
\hline Range & \multicolumn{2}{|c|}{$24-90$} \\
\hline Gender & Value & $\%$ \\
\hline Female & 42 & 41.18 \\
\hline Male & 60 & 58.82 \\
\hline Ethnicity & Value & $\%$ \\
\hline White (not Hispanic or Latino) & 86 & 84.31 \\
\hline White (Hispanic or Latino) & 4 & 3.92 \\
\hline White (ethnicity not reported) & 4 & 3.92 \\
\hline Asian & 7 & 6.86 \\
\hline Not reported & 1 & 0.98 \\
\hline Primary tumor site & Value & $\%$ \\
\hline Extremities & 40 & 39.22 \\
\hline Head and Neck & 7 & 6.86 \\
\hline Trunk & 46 & 45.10 \\
\hline Trunk and Extremities & 1 & 0.98 \\
\hline Other site & 4 & 3.92 \\
\hline Not reported & 4 & 3.92 \\
\hline Clark level at diagnosis & Value & $\%$ \\
\hline I - III & 15 & 14.71 \\
\hline IV - V & 57 & 55.88 \\
\hline Not reported & 30 & 29.41 \\
\hline \multicolumn{3}{|l|}{ Breslow thickness at diagnosis $(\mathrm{mm})$} \\
\hline Mean & \multicolumn{2}{|c|}{11.7136667} \\
\hline Median & \multicolumn{2}{|c|}{10} \\
\hline
\end{tabular}

Fig. 7 Low expression of TAK1 in primary melanoma samples is associated with favourable patient prognosis. a Cohort characteristics ( $n=102$ patients). b Difference in mean disease free survival (DFS) including the $95 \%$ confidence interval obtained by screening for a group separator for high and low MAP $3 K 7 \mathrm{mRNA}$ expression. The optimal separator is indicated by a vertical line. The distribution of

establish necroptosis competency in melanoma, highlighting that additional regulatory processes must play a role in suppressing necroptosis competency in this setting.

One obvious reason might be that in addition to insufficient RIPK3 also reduced MLKL expression limits necroptosis in melanoma. Indeed, MLKL expression correlated with necroptosis susceptibility in a large cell linebased screen [21], and we here found that MLKL is heterogeneously expressed in melanoma cell lines. However, we showed that melanoma cells expressing MLKL failed to undergo necroptosis, even in presence of elevated RIPK3 amounts. MLKL expression increased after 5-AD pretreatment in Malme-3M and MEL-JUSO cells, but these cells also remained necroptosis resistant. It is noteworthy, though, that the increase in MLKL after 5-AD treatment indicates that also MLKL expression could be silenced by DNA methylation. To our knowledge, this has not yet been
B
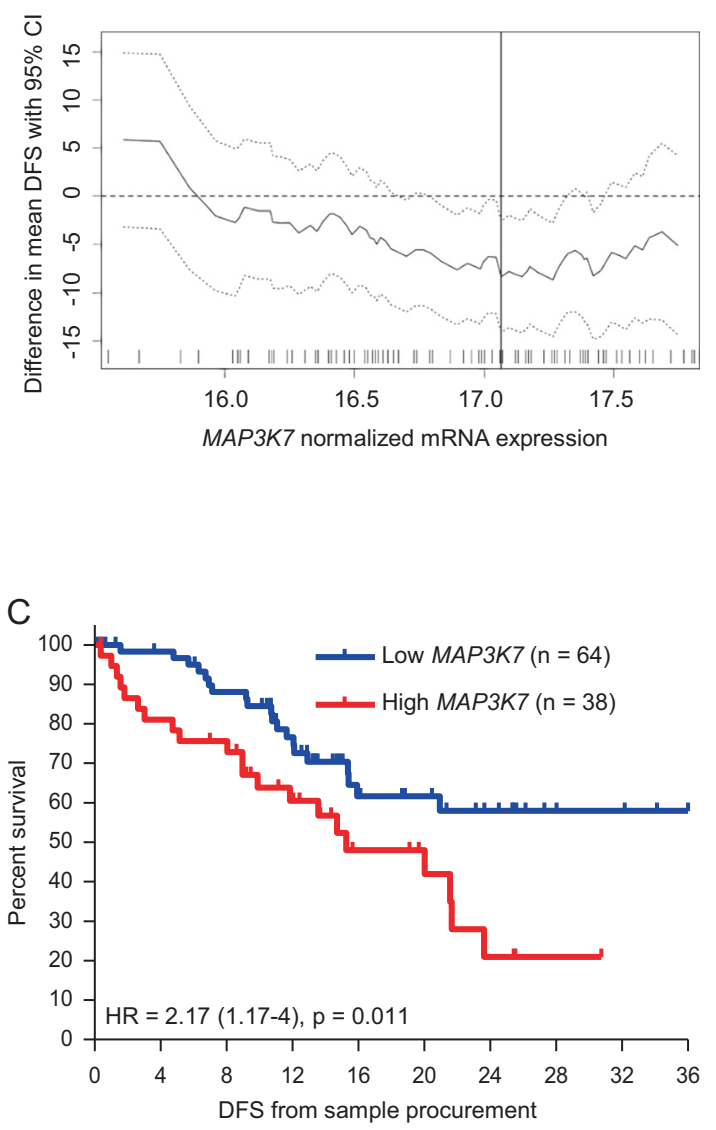

normalized expression of MAP3K7 mRNA $(\log 2(\mathrm{FPKM}-\mathrm{UQ}+1))$ is shown as a rug plot at the bottom of the graph. c Survival analysis in the SKCM-TCGA primary sub-cohort ( $n=102$ patients). Disease free survival (DFS) from sample procurement of patients with high (red) and low (blue) MAP $3 K 7$ mRNA expression was compared by log-rank test

studied, but would align with the more general hypothesis that disease progression in cancer might select for increased necroptosis resistance [21].

We found that signal transduction towards necroptosis never proceeds past RIPK1, indicating that additional regulatory processes must exist. We identified TAK1 as a critical regulator of cell death susceptibility in response to activating TNF receptors and inhibiting cellular IAPs. TAK1 is well known to modulate both apoptosis and necroptosis susceptibility [14-16], but the consequences of interfering with TAK1 activity appear to be complex and possibly context dependent. In necroptosis competent fibroblasts, the inhibition of TAK1 suppresses pro-survival $\mathrm{NF \kappa B}$ signaling in response to $\mathrm{TNF} \alpha$, augments RIPK1 phosphorylation, and thereby strengthens both apoptosis and necroptosis signaling branches [14]. Furthermore, the extent by which TAK1 directly phosphorylates RIPK1 can 
regulate apoptosis vs. necroptosis signaling and cell death. If NFKB signaling is suppressed, transient RIPK1 Ser321 phosphorylation supports RIPK1-independent apoptosis, whereas sustained multi-site phosphorylation of RIPK1 supports necroptosis in L929 cells and MEFs [15]. Irrespective of this and regardless of whether TAK1 is inhibited or not, it remains to be studied why melanoma cells exclusively direct signaling towards apoptosis rather than necroptosis, even though all key necroptosis effectors are in place and treatment scenarios otherwise sufficient to induce necroptosis were chosen. Of note, we observed in TRAIL treatment scenarios that cell death remained completely independent of RIPK1 activity, even though TAK1 can regulate TRAIL responsiveness $[39,40]$. During TRAIL-induced signaling, a cell deathsuppressing role of TAK1 might be less apparent since the apoptosis branch, in contrast to TNF $\alpha$-induced cell death, relies less on forming an intracellular complex II [41, 42]. Since TAK1 also regulates interleukin and toll-like receptor pathway signaling [43], interfering with the basal activities of these pathways through targeting TAK1 might have contributed to cell death sensitization in our studies.

We found that high TAK1 expression correlates with a higher risk of disease recurrence in patients diagnosed with primary melanoma. Since TNF $\alpha$ is found within melanoma microenvironments $[10,37]$, TAK1 might contribute to suppressing cell death in situ and thereby promote disease progression. Of note, TNF $\alpha$ is given in combination with DNA-alkylating agents to exert anti-tumor activity during the treatment of loco-regionally metastatic melanoma by isolated limb perfusion [44]. This treatment regimen is not tolerated systemically but clearly of benefit to many patients. Suppressing TAK1 activity with specific inhibitors [28] could, however, provide opportunities to improve these therapeutic strategies in the future.

\begin{abstract}
Acknowledgements We mourn the passing of our thoughtful and enthusiastic co-author, colleague and friend Martin Leverkus. LGP and MR kindly acknowledge support by the European Union's Horizon 2020 research and innovation programme under the Marie Skłodowska-Curie grant agreement \#642295 (MEL-PLEX). MR and DPD also receive support from the German Research Foundation (FOR2036 (MO 3226/1-1), DI 2440/3-1). MR receives further support from the European Union's Horizon 2020 research and innovation programme (grant agreements \#766069 (GLIO-TRAIN), \#675448 (TRAIN-ERS)) and the Health Research Board Ireland (HRA POR 2013 245, HRA POR 2015 1091).
\end{abstract}

\section{Compliance with ethical standards}

Conflict of interest The authors declare that they have no conflict of interest.

Publisher's note: Springer Nature remains neutral with regard to jurisdictional claims in published maps and institutional affiliations.

\section{References}

1. Robert C, Karaszewska B, Schachter J, Rutkowski P, Mackiewicz A, Stroiakovski D, et al. Improved overall survival in melanoma with combined dabrafenib and trametinib. $\mathrm{N}$ Engl $\mathrm{J}$ Med. 2015;372:30-39.

2. Luke JJ, Flaherty KT, Ribas A, Long GV. Targeted agents and immunotherapies: optimizing outcomes in melanoma. Nat Rev Clin Oncol. 2017;14:463-82.

3. Vanden Berghe T, Kaiser WJ, Bertrand MJ, Vandenabeele P. Molecular crosstalk between apoptosis, necroptosis, and survival signaling. Mol Cell Oncol. 2015;2:e975093.

4. Lafont E, Hartwig T, Walczak H. Paving TRAIL's path with ubiquitin. Trends Biochem Sci. 2018;43:44-60.

5. Taylor RC, Cullen SP, Martin SJ. Apoptosis: controlled demolition at the cellular level. Nat Rev Mol Cell Biol. 2008;9:231-41.

6. Galluzzi L, Kepp O, Chan FK, Kroemer G. Necroptosis: mechanisms and relevance to disease. Annu Rev Pathol. 2017;12:103-30.

7. Wang H, Sun L, Su L, Rizo J, Liu L, Wang LF, et al. Mixed lineage kinase domain-like protein MLKL causes necrotic membrane disruption upon phosphorylation by RIP3. Mol Cell. 2014;54:133-46.

8. Chan FK, Shisler J, Bixby JG, Felices M, Zheng L, Appel M, et al. A role for tumor necrosis factor receptor-2 and receptorinteracting protein in programmed necrosis and antiviral responses. J Biol Chem. 2003;278:51613-21.

9. Feng S, Yang Y, Mei Y, Ma L, Zhu DE, Hoti N, et al. Cleavage of RIP3 inactivates its caspase-independent apoptosis pathway by removal of kinase domain. Cell Signal. 2007;19:2056-67.

10. Smith MP, Sanchez-Laorden B, O'Brien K, Brunton H, Ferguson $\mathrm{J}$, Young $\mathrm{H}$, et al. The immune microenvironment confers resistance to MAPK pathway inhibitors through macrophage-derived TNFalpha. Cancer Discov. 2014;4:1214-29.

11. Bertrand F, Montfort A, Marcheteau E, Imbert C, Gilhodes J, Filleron T, et al. TNFalpha blockade overcomes resistance to antiPD-1 in experimental melanoma. Nat Commun. 2017;8:2256.

12. Geserick P, Hupe M, Moulin M, Wong WW, Feoktistova M, Kellert B, et al. Cellular IAPs inhibit a cryptic CD95-induced cell death by limiting RIP1 kinase recruitment. J Cell Biol. 2009; $187: 1037-54$.

13. Fulda S. Molecular pathways: targeting inhibitor of apoptosis proteins in cancer-from molecular mechanism to therapeutic application. Clin Cancer Res. 2014;20:289-95.

14. Guo X, Yin H, Chen Y, Li L, Li J, Liu Q. TAK1 regulates caspase 8 activation and necroptotic signaling via multiple cell death checkpoints. Cell death Dis. 2016;7:e2381.

15. Geng J, Ito Y, Shi L, Amin P, Chu J, Ouchida AT, et al. Regulation of RIPK1 activation by TAK1-mediated phosphorylation dictates apoptosis and necroptosis. Nat Commun. 2017;8:359.

16. Mihaly SR, Ninomiya-Tsuji J, Morioka S. TAK1 control of cell death. Cell Death Differ. 2014;21:1667-76.

17. Geserick P, Wang J, Schilling R, Horn S, Harris PA, Bertin J, et al. Absence of RIPK3 predicts necroptosis resistance in malignant melanoma. Cell death Dis. 2015;6:e1884.

18. Ninomiya-Tsuji J, Kishimoto K, Hiyama A, Inoue J, Cao Z, Matsumoto K. The kinase TAK1 can activate the NIK-I kappaB as well as the MAP kinase cascade in the IL-1 signalling pathway. Nature. 1999;398:252-6.

19. Cerami E, Gao J, Dogrusoz U, Gross BE, Sumer SO, Aksoy BA, et al. The cBio cancer genomics portal: an open platform for exploring multidimensional cancer genomics data. Cancer Discov. 2012;2:401-4.

20. Budczies J, Klauschen F, Sinn BV, Gyorffy B, Schmitt WD, Darb-Esfahani $S$, et al. Cutoff finder: a comprehensive and 
straightforward Web application enabling rapid biomarker cutoff optimization. PLoS ONE. 2012;7:e51862.

21. Najafov A, Zervantonakis IK, Mookhtiar AK, Greninger P, March RJ, Egan RK, et al. BRAF and AXL oncogenes drive RIPK3 expression loss in cancer. PLoS Biol. 2018;16:e2005756.

22. Koo GB, Morgan MJ, Lee DG, Kim WJ, Yoon JH, Koo JS, et al. Methylation-dependent loss of RIP3 expression in cancer represses programmed necrosis in response to chemotherapeutics. Cell Res. 2015;25:707-25.

23. Feoktistova M, Wallberg F, Tenev T, Geserick P, Leverkus M, Meier P. Techniques to distinguish apoptosis from necroptosis. Cold Spring Harb Protoc. 2016;2016:pdbtop070375.

24. Hutt M, Marquardt L, Seifert O, Siegemund M, Muller I, Kulms $\mathrm{D}$, et al. Superior properties of Fc-comprising scTRAIL fusion proteins. Mol Cancer Ther. 2017;16:2792-802.

25. Yang Z, Jiang B, Wang Y, Ni H, Zhang J, Xia J, et al. 2-HG inhibits necroptosis by stimulating DNMT1-dependent hypermethylation of the RIP3 promoter. Cell Rep. 2017;19:1846-57.

26. Patel K, Dickson J, Din S, Macleod K, Jodrell D, Ramsahoye B. Targeting of 5-aza-2'-deoxycytidine residues by chromatinassociated DNMT1 induces proteasomal degradation of the free enzyme. Nucleic Acids Res. 2010;38:4313-24.

27. Wu J, Powell F, Larsen NA, Lai Z, Byth KF, Read J, et al. Mechanism and in vitro pharmacology of TAK1 inhibition by (5Z)-7-oxozeaenol. ACS Chem Biol. 2013;8:643-50.

28. Kilty I, Jones LH. TAK1 selective inhibition: state of the art and future opportunities. Future Med Chem. 2015;7:23-33.

29. Schadendorf D, Moller A, Algermissen B, Worm M, Sticherling M, Czarnetzki BM. IL-8 produced by human malignant melanoma cells in vitro is an essential autocrine growth factor. J Immunol. 1993;151:2667-75.

30. Bar-Eli M. Role of interleukin-8 in tumor growth and metastasis of human melanoma. Pathobiology. 1999;67:12-18.

31. Richmond A, Thomas HG. Melanoma growth stimulatory activity: isolation from human melanoma tumors and characterization of tissue distribution. J Cell Biochem. 1988;36:185-98.

32. Dhawan P, Richmond A. Role of CXCL1 in tumorigenesis of melanoma. J Leukoc Biol. 2002;72:9-18.

33. Payne AS, Cornelius LA. The role of chemokines in melanoma tumor growth and metastasis. J Invest Dermatol. 2002;118:915-22.
34. Stove C, Vanrobaeys F, Devreese B, Van Beeumen J, Mareel M, Bracke M. Melanoma cells secrete follistatin, an antagonist of activin-mediated growth inhibition. Oncogene. 2004;23:5330-9.

35. Becker JC, Dummer R, Hartmann AA, Burg G, Schmidt RE. Shedding of ICAM-1 from human melanoma cell lines induced by IFN-gamma and tumor necrosis factor-alpha. Functional consequences on cell-mediated cytotoxicity. J Immunol. 1991;147:4398-401.

36. Dondelinger Y, Aguileta MA, Goossens V, Dubuisson C, Grootjans S, Dejardin E, et al. RIPK3 contributes to TNFR1mediated RIPK1 kinase-dependent apoptosis in conditions of cIAP1/2 depletion or TAK1 kinase inhibition. Cell Death Differ. 2013;20:1381-92.

37. Bergenwald C, Westermark G, Sander B. Variable expression of tumor necrosis factor alpha in human malignant melanoma localized by in situ hybridization for mRNA. Cancer Immunol Immunother. 1997;44:335-40.

38. Santoro R, Carbone C, Piro G, Chiao PJ, Melisi D. TAK-ing aim at chemoresistance: The emerging role of MAP3K7 as a target for cancer therapy. Drug Resist Updat. 2017;33-35:36-42.

39. Herrero-Martin G, Hoyer-Hansen M, Garcia-Garcia C, Fumarola C, Farkas T, Lopez-Rivas A, et al. TAK1 activates AMPKdependent cytoprotective autophagy in TRAIL-treated epithelial cells. EMBO J. 2009;28:677-85.

40. Morioka S, Omori E, Kajino T, Kajino-Sakamoto R, Matsumoto K, Ninomiya-Tsuji J. TAK1 kinase determines TRAIL sensitivity by modulating reactive oxygen species and cIAP. Oncogene. 2009;28:2257-65.

41. Lafont E, Kantari-Mimoun C, Draber P, De Miguel D, Hartwig T, Reichert M, et al. The linear ubiquitin chain assembly complex regulates TRAIL-induced gene activation and cell death. EMBO J. 2017;36:1147-66

42. Jin Z, El-Deiry WS. Distinct signaling pathways in TRAIL-versus tumor necrosis factor-induced apoptosis. Mol Cell Biol. 2006;26:8136-48.

43. Sakurai H. Targeting of TAK1 in inflammatory disorders and cancer. Trends Pharmacol Sci. 2012;33:522-30.

44. van Horssen R, Ten Hagen TL, Eggermont AM. TNF-alpha in cancer treatment: molecular insights, antitumor effects, and clinical utility. Oncologist. 2006;11:397-408. 